\title{
CONSUMO DE NUTRIENTES E BALANÇO HÍDRICO EM EQUINOS RECEBENDO DIETAS COM DIFERENTES NÍVEIS DE INCLUSÃO DE FENO DE ALFAFA
}

\author{
Leonir Bueno Ribeiro1, Carlos Eduardo Furtado2, Roberta Ariboni Brandi3, Andréa Cristina Souza \\ Paula4, Cleiton Luiz Tonello5, Daniel Augusto Afonso6
}

1- Mestre em Zootecnia, UEM/ Maringá. - leonirbueno@hotmail.com

2 - Professor Doutor da Universidade Estadual de Maringá - UEM. DZO - Departamento de Zootecnia.

3 - Professora Doutora do Departamento de Zootecnia - FZEA/USP

4 - Zootecnista, Universidade Estadual de Maringá - UEM

5 - Zootecnista, Escola Superior de Agronomia de Paraguaçu Paulista - ESAPP

6 - Aluno do curso de Zootecnia da Escola Superior de Agronomia de Paraguaçu Paulista - ESAPP

\section{RESUMO}

Objetivou-se avaliar o consumo de nutrientes e o balanço hídrico em equinos em mantença, alimentados com fenos convencionais. Utilizaram-se seis equinos machos com idade média de nove anos e PV de $361,16 \pm 12 \mathrm{~kg}$, em delineamento quadrado latino $(6 \times 6)$, alojados em gaiolas de metabolismo. Os tratamentos foram FTT: 100\% Feno de Tifton, FTO: $80 \%$ Feno de Tifton $+20 \%$ de Feno de Alfafa, FTS: $60 \%$ Feno de Tifton $+40 \%$ de Feno de Alfafa, FTQ: $40 \%$ Feno de Tifton $+60 \%$ de Feno de Alfafa, FTV: $20 \%$ Feno de Tifton $+80 \%$ de Feno de Alfafa, FAT: 100\% de Feno de Alfafa. As quantidades de alimentos fornecidos foram de 2,25\% do PV, obtendo-se sobras. Para o consumo de água, utilizaram-se bebedouros com forneci- mento de água à vontade. Os consumos de PB, EE e MM aumentaram à medida que o feno de alfafa foi incrementado nas dietas indicando que esses elementos estão relacionados à composição química das dietas. Os valores de consumo e de excreção de água foram influenciados pela composição química da dieta, influenciando diretamente no consumo de água em relação à matéria seca (litros $/ \mathrm{kg}$ de MSI) e no balanço hídrico. Com relação ao consumo e excreção de água, observou-se que em todos os tratamentos os animais apresentaram-se em balanço hídrico positivo. O tratamento FTT apresentou uma menor excreção fecal e urinária de água em relação aos demais tratamentos.

PALAVRAS-CHAVE: Alimentação; cavalos; hidratação; nutrição.

\section{NUTRIENTS INTAKE AND WATER BALANCE OF EQUINE FED DIETS WITH DIFFERENT LEVELS OF INCLUSION OF ALFALFA HAY}

The objective of this study was to evaluate the nutrients intake and water balance of horses in maintenance, fed with conventional hay. Six male horses with an average age of nine years and live weight of $361.16 \pm 12 \mathrm{~kg}$ were 
used, in a latin square design (6x6), housed in metabolism cages. The treatments were FTT: $100 \%$ Hay of Tifton, FTO: $80 \%$ Hay of Tifton $+20 \%$ Hay of Alfalfa, FTS: $60 \%$ Hay of Tifton $+40 \%$ Hay of Alfalfa, FTQ: $40 \%$ Hay of Tifton $+60 \%$ Hay of Alfalfa, FTV: $20 \%$ Hay of Tifton $+80 \%$ Hay of Alfalfa, FAT: $100 \%$ Hay of Alfalfa. The quantities of food provided were $2.25 \%$ of BW in order to obtain surplus. To determine the water intake, drinkers with water supply ad libitum were used. The intakes of DM, CP, EE and MM increased $(\mathrm{P} \leq 0.05)$ as alfalfa hay amount was increased in the diets,

KEYWORDS: Feeding; horse; hydration; nutrition

\section{INTRODUÇÃO}

Em algumas provas equestres como enduro e as marchas, nas quais o animal percorre longas distâncias, o aporte de nutrientes e de eletrólitos mantidos em seu trato digestório é fundamental para que o animal possa concluir eficientemente as provas. Muitos nutricionistas recomendam o uso de fenos de gramíneas ao invés do tradicional feno de alfafa, pois este apresenta teor proteico que possibilita maior gasto energético para realizar o metabolismo proteico e, consequentemente, maior excreção de água. Aproximadamente $90 \%$ da perda de peso representam mudanças no balanço dos fluídos, sendo a perda de peso durante as provas de enduro largamente atribuída à desidratação (LEWIS, 2000; NRC, 2007).

Dentre os nutrientes requeridos, a água, muitas vezes por ser "abundante e barata", é negligenciada e, segundo NYMAN \& DAHLBORN (2000), os equinos submetidos ao esforço físico têm grande aumento na produção de calor devido à ineficiência do metabolismo energético. Logo, observa-se em animais que realizam exercícios de moderada e alta intensidade de 40 a 60 vezes mais calor que o produzido pelo metabolismo basal, sendo a sudorese a principal via de dispersão desse calor nos equinos em que o volume de suor depende em parte da intensidade do exercício, da distância, do tipo de andamento, do terreno e da quantidade de peso carregado, influenciando diretamente no consumo de água (EATON, 1994; ERIKSON, 1996; GOER et al. 2000). No entanto, é difícil predizer as necessidades de consumo de água em cavalos de competição (NRC, 2007).

Desta forma, objetivou-se avaliar o consumo de nutrientes e o balanço hídrico em equinos adultos, quando submetidos a dietas de fenos convencionais indicating that these elements are related to the diets chemical composition. Similarly, NDF and ADF intake decreased $(P \leq 0.05)$ as alfalfa hay was increased in the diets. The values of water intake and excretion were influenced by the chemical composition of the diet, influencing water intake in function of the dry matter (liters/ $\mathrm{kg}$ of DM) and the water balance. Regarding water intake and excretion, the animals were in positive water balance in all treatments. The FTT treatment had a lower urinary and fecal excretion of water compared to the other treatments.

com diferentes níveis de inclusão de feno de alfafa.

\section{MATERIAL E MÉTODOS}

O experimento foi conduzido no setor de Equideocultura da Fazenda Experimental de Iguatemi, da Universidade Estadual de Maringá-UEM. Utilizaram-se seis equinos machos mestiços com idade média de nove anos e peso vivo de $361,16 \pm 12 \mathrm{~kg}$, em delineamento experimental em quadrado latino (6x6), alojados em gaiolas de metabolismo.

Os tratamentos foram constituídos por seis dietas experimentais de diferentes proporções de feno de tifton 85 (Cynodon dactylon) e feno de alfafa (Medigaco sativa sp.), sendo FTT: 100\% Feno de Tifton, FTO: $80 \%$ Feno de Tifton $+20 \%$ de Feno de Alfafa, FTS: $60 \%$ Feno de Tifton $+40 \%$ de Feno de Alfafa, FTQ: $40 \%$ Feno de Tifton $+60 \%$ de Feno de Alfafa, FTV: $20 \%$ Feno de Tifton $+80 \%$ de Feno de Alfafa, FAT: $100 \%$ de Feno de Alfafa. As quantidades de alimentos fornecidos foram da ordem de 2,25 $\%$ do PV dos animais distribuídos em três refeições diárias $(8: 00 ; 15: 00$ e 21:00) de forma à obtenção de sobras, permitindo a realização dos cálculos de consumo. As análises químicas das amostras das dietas foram realizadas conforme metodologia e técnicas descritas por AOAC (1990), SILVA (2002) e VAN SOEST (1991) e estão apresentadas na Tabela 1.

O período experimental teve duração total de 66 dias, sendo sete dias de adaptação às dietas e quatro dias para a coleta dos dados. Para determinar o consumo de água, o volume foi obtido com uso de bebedouros graduados (tipo balde) e com fornecimento de água à vontade. As temperaturas do ambiente foram mensuradas com uso de termômetro (tipo coluna de mercúrio), concomitantemente ao fornecimento das dietas, obtendo-se média de $20,3^{\circ} \mathrm{C}$. Os 
dados obtidos foram avaliados por meio de análise de variância e as médias comparadas pelo teste de Tukey a $5 \%$ de probabilidade, usando-se o programa computacional Sistema de Análises Estatísticas e Genéticas - versão 9.1 (UNIVERSIDADE FEDERAL DE VIÇOSA, 2008)

TABELA 1. Composição química das dietas experimentais expressa em (\%) Matéria Seca

\begin{tabular}{lcccccc}
\hline \multicolumn{1}{c}{ Nutrientes ${ }^{1}(\%)$} & \multicolumn{7}{c}{ Tratamentos } \\
\hline Matéria Seca & FTT & FTO & FTS & FTQ & FTV & FAT \\
Matéria Orgânica & 81,1 & 91,02 & 90,94 & 90,86 & 90,78 & 90,69 \\
Extrato Etéreo & 1,01 & 86,21 & 85,56 & 84,91 & 84,25 & 83,6 \\
Proteína Bruta & 4,93 & 1,02 & 1,02 & 1,03 & 1,04 & 1,05 \\
FDN & 73,87 & 68,31 & 9,75 & 12,16 & 14,58 & 16,99 \\
FDA & 37,1 & 36,05 & 35,76 & 57,2 & 51,64 & 46,09 \\
Matéria Mineral & 4,35 & 4,9 & 5,45 & 33,95 & 32,91 & 31,86 \\
\hline
\end{tabular}

1- Analise realizada no laboratório de Nutrição Animal do Curso de Zootecnia - UEM

\section{RESULTADOS E DISCUSSÃO}

Observa-se na Tabela 2 que, consumo de matéria seca (CMS), o consumo de proteína bruta (CPB), o consumo de extrato etéreo (CEE) e o consumo de matéria mineral (CMM) aumentaram $(\mathrm{P} \leq 0,05)$ à medida que o feno de alfafa foi incrementado nas dietas, indicando que os valores obtidos podem estar relacionados principalmente com a composição química das dietas (PB, FDN e
FDA), sustentado pela similaridade dos teores de matéria seca entre os tratamentos (FRAPE, 1998; LEWIS, 2000; NRC, 2007). Os equinos podem aumentar o consumo de matéria seca, no intuito de atender às necessidades energéticas; contudo, a inclusão de óleo pode tornar dietas mais palatáveis e aumentar as densidades energéticas, além das características dos alimentos em teores energéticos e densidades (EATON, 1994; NRC, 2007).

TABELA 2. Consumo de nutrientes (gramas/dia) de equinos alimentados com feno de tifton e feno de alfafa

\begin{tabular}{|c|c|c|c|c|c|c|c|}
\hline \multirow[t]{2}{*}{ Variáveis } & \multicolumn{6}{|c|}{ Tratamentos } & \multirow[t]{2}{*}{ CV\% } \\
\hline & FTT & FTO & FTS & FTQ & FTV & FAT & \\
\hline CMS & $5602,55^{\circ}$ & $6672,32^{a}$ & $7214,21^{a}$ & 6964,27 & $7056,31^{*}$ & 7038,97 & 7,98 \\
\hline CPB & 275,97 & $489,05^{e}$ & $702,50^{\mathrm{d}}$ & $846,17^{c}$ & $1027,89^{b}$ & $1195,79^{\mathrm{a}}$ & 7,13 \\
\hline CEE & $62,09 \mathrm{~b}$ & 74,57 & $81,29 \mathrm{a}$ & $79,12^{\mathrm{a}}$ & $80,82^{\mathrm{a}}$ & 81,27 & 7,86 \\
\hline CFDN & $4138,48^{\mathrm{sb}}$ & $4559,33^{\mathrm{a}}$ & $4529,52^{\mathrm{a}}$ & $3985,67^{\mathrm{sb}}$ & $3645,60^{\text {bc }}$ & $3244,14^{c}$ & 9,59 \\
\hline CFDA & $2078,28^{b}$ & $2405,47^{a}$ & $2525,40^{2}$ & $2364,95^{\mathrm{sb}}$ & $2322,16^{\mathrm{ob}}$ & $2242,45^{\mathrm{ab}}$ & 8,45 \\
\hline $\mathrm{CMM}$ & $243,44^{*}$ & $326,80^{4}$ & $393,28^{c}$ & $418,29 c$ & $463,03^{\mathrm{sb}}$ & $501,0 \mathrm{P}$ & 6,99 \\
\hline
\end{tabular}

Médias seguidas de mesma letra na linha não diferem estatisticamente pelo teste de Tukey em nível de probabilidade de 5\%

Por outro lado, o consumo de FDN e FDA diminuiu $(\mathrm{P} \leq 0,05)$ à medida que o feno de alfafa foi incrementado nas dietas. Para os tratamentos com maior nível de feno de tifton observou-se menor consumo de nutrientes, indicando que os equinos podem aumentar o consumo de matéria seca no intuído de atender às exigências nutricionais; contudo, ficam limitados pelos teores da fração fibrosa da di- eta (FDN e FDA).

O consumo de matéria seca do presente trabalho variou entre os tratamentos de 1,52 a $1,98 \%$ PV. Segundo o NRC (1989), os equinos consomem diariamente de 1,5 a $3,0 \%$ de seu peso vivo na base da matéria seca dependo da quantidade disponível, qualidade e estágio fisiológico das plantas. Quando a exigência energética do animal não é atendida pe- 
lo aporte dietético exclusivo a partir de forrageiras em regime "ad libitum", recomenda-se no mínimo $1,0 \%$ do PV de volumosos e a suplementação com concentrado. O NRC (2007) recomenda para animais em mantença consumo de matéria seca de 2,0 $\% \mathrm{PV}$.

A Tabela 3 apresenta em litros/dia o consumo de água (CAG), consumo de água da dieta (CAD), relação entre litros por $\mathrm{kg}$ de matéria seca ingerida (RLM), excreção fecal de água (EFA), excreção urinária de água (EUA) e o balanço hídrico (BAH). Os valores de $\mathrm{CAG}, \mathrm{CAD}, \mathrm{EFA}$ e EUA aumentaram $(\mathrm{P} \leq 0,05)$ à medida que o feno de alfafa foi incre- mentado nas dietas. O consumo de água $(\mathrm{CAG}+$ CAD) e a excreção de água (EFA + EUA) foram influenciados tanto pela composição química das dietas (PB, FDN e FDA) como pelo consumo de nutrientes (MS, PB, FDN e FDA), justificado pelo arraste de fluídos pela fração fibrosa nas fezes (FRAPE, 1998). Apesar de a excreção de nitrogênio não ter sido mensurada no presente trabalho, sugerese que o maior consumo de água nas dietas com nível crescente de feno de alfafa possa estar relacionado com a maior necessidade de excreção nitrogenada (NRC, 2007).

TABELA 3. Parâmetros associados à relação de água por kg de matéria seca ingerida e ao balanço hídrico (litros/dia) de equinos consumindo feno de tifton e feno de alfafa

\begin{tabular}{|c|c|c|c|c|c|c|c|}
\hline \multirow{2}{*}{ Variáveis } & \multicolumn{6}{|c|}{ Tratamentos } & \multirow{2}{*}{ CV\% } \\
\hline & FTT & FTO & FTS & FTQ & FTV & FAT & \\
\hline CAG & $22,08^{b}$ & $26,08^{\mathrm{ab}}$ & $28,6 \mathrm{~Pb}$ & $26,71^{2 b}$ & $24,79^{\mathrm{b} b}$ & $32,42^{\mathrm{a}}$ & 17,78 \\
\hline $\mathrm{CAD}$ & $0,547^{\circ}$ & $0,658^{\circ}$ & $0,719^{a}$ & $0,701^{\circ}$ & 0,717 & $0,722^{\circ}$ & 7,84 \\
\hline RLM & $4,06^{\mathrm{ab}}$ & $3,93^{b}$ & $3,96^{\mathrm{b}}$ & $3,82^{b}$ & $3,48^{b}$ & $4,61^{\mathrm{a}}$ & 9,13 \\
\hline EFA & $10,96^{c}$ & $13,73^{\mathrm{ab}}$ & $15,08^{\mathrm{a}}$ & 12,770 & $13,83^{b b}$ & $12,28^{\mathrm{bc}}$ & 7,48 \\
\hline EUA & $2,50^{\circ}$ & $3,16^{\mathrm{cd}}$ & $3,96^{\mathrm{cd}}$ & $5,48^{b c}$ & $7,00^{\mathrm{sb}}$ & $8,83^{\mathrm{a}}$ & 25,12 \\
\hline $\mathrm{BAH}$ & 9,170 & $9,85^{\circ}$ & $10,34^{\circ}$ & $9,16^{\mathrm{b}}$ & $9,63^{\circ}$ & $12,03^{a}$ & 18,45 \\
\hline
\end{tabular}

Médias seguidas de mesma letra na linha não diferem estatisticamente pelo teste de Tukey em nível de probabilidade de 1\%

Para o consumo de água (CAG), observa-se que houve diferença significativa entre os tratamentos, sendo que o tratamento FAT apresentou o maior valor, 32,42 litros/dia, seguido dos tratamentos FTO, FTS, FTQ, FTV, com valor médio de 26,55 litros/dia. Os valores observados no presente estudo estão acima dos encontrados por GALZERANO et at. (2006) e BRETAS et al. (2007) para dietas composta de tifton e tifton associado a alfafa, que encontraram 13,13 e 19,24 litros/ dia, respectivamente.

O consumo de água pelos equinos pode estar relacionado principalmente com o teor de matéria seca e proteico das dietas às quais os animais estão sendo submetidos e, observando-se a Tabela 1, pode-se inferir que os animais tiveram um menor consumo de nitrogênio quando alimentados com a dieta FTT em relação às demais, o que pode ser devido à maior necessidade de excreção de nitrogênio. Segundo OLIVEIRA et al. (2003), o metabólito de maior excreção na urina é a uréia proveniente do metabolismo hepático da amônia absorvida no intestino grosso e oriunda do catabolismo dos aminoácidos e, para que haja condições metabólicas para tal, consumos maiores de água são necessários. FONNESBECK (1968) observou o consumo de água à vontade durante ensaios de metabolismo com cavalos em mantença, sugerindo a possibilidade de realização de equações de regressão que expliquem o consumo de água em função da matéria seca ingerida, com grau elevado de confiança.

Com relação ao consumo de água da dieta (CAD), houve diferenças significativas somente no tratamento FTT, no qual se observou o menor valor de 0,547 litros/dia. Os demais não se diferenciaram estatisticamente, apresentando valor médio de 0,703 litros/dia. A ingestão de água da dieta está relacionada com o teor de matéria seca ou o total de alimento ingerido, assim como o consumo de água também pode variar com o tipo de processamento do volumoso (triturado, moído, peletizado) e com a forma de conservação (in natura, silagem, feno), isso porque a concentração dos nutrientes (proteína, minerais, teor fibroso e de outros componentes) é diferente entre os alimentos e suas formas de apresentação, afetando o consumo de água (CUNHA, 1991; GALZERANO et al. 2006; BRETAS et al. 
2007; NRC, 2007).

OLIVEIRA et al. (2003) relataram um consumo diário de água, com média de 3,88 litros $/ \mathrm{kg}$ de MSI, fornecendo dietas com diferentes relações de concentrado:volumoso para equinos. Segundo o NRC (2007), animais consumindo dietas completas apresentam média de 2,9 L/kg MSI, recomendandose o consumo mínimo de 2 a $3 \mathrm{~L} / \mathrm{kg}$ MSI.

NYMAN \& DAHLBORN (2000) relataram valores de ingestão de água em equinos alimentados com dietas mistas de feno e aveia, entre 0,043 e $0,058 \mathrm{~L} / \mathrm{kg} \mathrm{PV}$, valores inferiores aos obtidos no presente trabalho. CYMBALUK (1989) relatou consumo de 2,7 a 5,5 litros de água/100 kg de PV para cavalos mantidos em baias sob temperaturas moderadas. GOER et al. (2000), trabalhando com cavalos de peso médio de $500 \mathrm{~kg}$, em atividade física moderada, relataram um consumo de água em 8,2 L/ 100 kg de PV.

Para a relação entre consumo de água em litros por $\mathrm{kg}$ de matéria seca ingerida (RLM), observa-se que houve diferença estatística $(\mathrm{P} \leq 0,05)$ entre os tratamentos, sendo que o tratamento FAT apresentou o maior valor de 4,61 L/ $\mathrm{kg}$ de MSI, seguido pelo tratamento FTT com 4,06 L/ kg de MSI. Os demais tratamentos não se diferenciaram entre si apresentando um valor médio de 3,79 L/ kg de MSI. OLIVEIRA et al. (2003) relataram consumo diário de água com média de 3,88 litros/kg de MSI, fornecendo dietas com diferentes relações de concentrado:volumoso para equinos. Segundo o NRC (2007), animais consumindo dietas exclusivas de feno ingerem água em uma proporção de 3,6 litros de água por $\mathrm{kg}$ de MSI, enquanto que, para os animais alimentados com dietas compostas por feno e concentrado, essa proporção é de 2,9:1, recomendando-se o consumo de 2 a 3 litros de água/kg de MSI.

FRAPE (1998) destaca que o consumo de água por equinos alimentos com fontes de feno esteja entre 2 a 4 litros $/ \mathrm{kg}$ MSI. O consumo de água por $\mathrm{kg}$ de matéria seca ingerida observado no presente trabalho encontra-se um pouco acima; porém, é semelhante aos valores de consumo encontrados por BRETAS et al. (2007) de 2,56 e 3,91 L/ kg de MSI, respectivamente para dietas exclusiva de tifton e tifton associado a alfafa. Destaca-se que o valor encontrado por BRETAS et al. (2007), para dietas com tifton associadas com alfafa, é semelhante à média encontrada no presente trabalho para os demais tratamentos (FTO, FTS, FTQ e FTV) que não apresen- taram diferenças significativas, obtendo-se valor médio de 3,79 L/ $\mathrm{kg}$ de MSI. GALZERANO et al. (2006) e BRETAS et al. (2007), em seus estudos obtiveram valores superiores aos observados por PEARSON MERRITT (1991), no qual encontrou consumo de 1,92 litros/kg de MSI; no entanto, os valores em geral estão de acordo com CYMBALUK (1989), que encontrou valores de 3,21 e 3,42 litros/kg de MSI, respectivamente.

A quantidade de água consumida e consequentemente excretada pelos equinos está relacionada a diversos fatores, como já destacado anteriormente, como a composição química dos alimentos associada especialmente ao conteúdo de proteínas, minerais e fibras; porém, a digestibilidade das dietas, a temperatura e a umidade relativa do ar, a atividade física e o estágio fisiológico em que se encontram os animais são vetores cumulativos sobre as perdas e têm que ser compensadas pela ingestão de água (CYMBALUK, 1989; KRISTULA \& McDONNELL, 1994; McDONNELL \& KRISTULA, 1996).

Segundo McDONNELL et al. (1999), equinos estabulados consumindo água em baldes, possivelmente por exercer um estimulo físico e compulsivo ao consumo, podem apresentar um aumento de ingestão de água em até $30 \%$ em relação a animais recebendo água em bebedouros automáticos, o que poderia explicar, dentre outros aspectos já citados, um possível aumento no consumo de água por $\mathrm{kg}$ de matéria seca ingerida, em relação aos demais autores.

Com relação à excreção fecal de água (EFA), pôde-se observar que houve diferenças significativas, sendo que o tratamento que apresentou o maior valor foi FTS com 15,08 litros/dia, seguido pelo tratamento FTO com 13,73 litros/dia. Os valores encontrados no presente trabalho foram superiores aos encontrados por BRETAS et al. (2007) que, trabalhando com equinos alimentados com dietas de tifton e tifton associado a alfafa, encontrou valores de 4,28 e 7,93 litros/dia, respectivamente. OLIVEIRA et al. (2003), relataram que, avaliando dietas para equinos com níveis crescentes de feno de coast cross, o nível de volumoso na dieta não afetou o consumo de água. No entanto, a excreção de água nas fezes foi aumentada com a inclusão dietética do volumoso, influenciando o balanço hídrico. Observando os valores apresentados na Tabela 2, para CMS, CFDN e CFDA, para os tratamentos FTO e 
FTS, e comparando com os valores apresentados na Tabela 3 para os mesmos tratamentos, pode-se observar que os maiores valores de consumo de matéria seca e consumo de fração fibrosa (FDN e FDA) foram obtidos pelos tratamentos que apresentaram maior excreção fecal de água. Dessa forma, pode se relacionar os maiores valores de excreção de água via fezes à composição da dieta. CYMBALUK (1989) relata em seu estudo que os maiores valores de excreção fecal de água se dão em dietas compostas com fenos de gramíneas.

FONNESBECK (1968) relata que a excreção de água pelas fezes é a principal via de excreção pelos equinos; porém, GROENENDYK et al. (1988) demonstraram que equinos consumindo feno de alfafa excretam principalmente pela urina. Isso indica que os valores obtidos estejam relacionados principalmente com a composição química das dietas (PB, FDN e FDA) e seus respectivos consumos (CMS, CPB, CFDN e CFDA), sustentados pela similaridade dos teores de MS entre os tratamentos (FRAPE, 1998; LEWIS, 2000; NRC, 2007).

Para a excreção urinaria de água (EUA), observou-se diferença $(P \leq 0,05)$ entre os tratamentos, sendo que o tratamento FAT apresentou o maior valor de 8,83 litros/dia. O menor valor observado foi com o tratamento FTT, que se apresentou com 2,50 litros/dia. Os valores apresentados seguem o padrão descrito por FONNESBECK (1968) e GROENENDYK et al. (1988).

Com relação ao consumo e excreção de água, observou-se que em todos os tratamentos os animais apresentaram-se em balanço hídrico (BAH) positivo. O tratamento FAT foi o que apresentou maior valor de balanço hídrico (12,03 litros/dia); por outro lado, observou-se que esse tratamento apresentou maior valor de excreção urinária de água, consequentemente, maior consumo de água (litros), consumo de água (litros) por kg de MSI, sugerindo que FAT ou dietas com teores maiores de proteína bruta possam induzir a uma perda maior de eletrólitos se o consumo de água não for adequado e atendido, possivelmente pela necessidade de metabolização da fração protéica da dieta (LEWIS, 2000).

CYMBALUK (1989), avaliando equinos alimentados com dietas constituídas exclusivamente por feno de gramínea ou leguminosa e/ou associados à ração concentrada, observou valores de excreção de água nas fezes próximos aos observados neste estudo. Tais valores referem-se ao aumento da participação da fração fibrosa e fração protéica nas dietas.

OLIVEIRA et al. (2003), avaliando cavalos alimentados com dietas exclusivas de feno, observou em seus estudos a maior ingestão de água, em comparação aos animais alimentados com dietas mistas. Dietas com altos níveis de fibra podem desenvolver um tempo menor de retenção no trato gastrintestinal, o que pode levar aos aumentos no nível de perdas de água nas fezes, em consequência, um aumento no consumo de água (RIBEIRO et al., 2009).

\section{CONCLUSÕES}

Os consumos de PB, EE e MM aumentaram à medida que o feno de alfafa foi incrementado nas dietas, indicando que esses valores estejam relacionados à composição química das dietas. Os valores de consumo e de excreção de água foram influenciados pela composição química da dieta, influenciando diretamente no consumo de água em relação à matéria seca (litros/kg de MSI) e influenciando no balanço hídrico. Com relação ao consumo e excreção de água, observou-se que em todos os tratamentos os animais apresentaram-se em balanço hídrico positivo. O tratamento FTT apresentou uma menor excreção fecal de água e menor excreção urinaria de água em relação aos demais tratamentos, sendo o FAT o tratamento que apresentou maiores valores para ambos os parâmetros.

\section{REFERÊNCIAS}

ASSOCIATION OF OFFICIAL ANALYTICAL CHEMISTS - AOAC. Official Methods of Analysis, 15. ed. Arlington: Virginia, 1990.1117p.

BRETAS, A.A.; ALMEIDA, F.Q.; VIEIRA, A.A.; ALMEIDA, M.I.V.; PIMENTEL, R.R.M.; SILVA, V.P.; SANTOS, T.M.; GODOI, F.N.; GALZERANO, L. Balanço hídrico e de nitrogênio em equinos alimentos com feno de alfafa, feno de tifton-85 e concentrado. Revista Brasileira de Ciência Veterinária, v.14, n.3, p.150-154, 2007.

CYMBALUK, N.F. Water balance of horses fed various diets. Equine Practice, v.11, n.1, p.19-24, 1989.

EATON, M. D. Energetic and Performance. In: _. The Athletic Horse: principles and practice of equine sports medicine, W. B. Sauders Company, Philadelphia, p. 4962. 1994. 
ERIKSON, H. H. Fisiologia do Exercício; In: _. Fisiologia dos Animais Domésticos, $11^{\mathrm{a}}$ ed., Rio de Janeiro, Guanabara Koogan S.A., p. 277-296. 1996.

FONNESBECK, P.V. Consumption and excretion of water by horses receiving all hay and hay-grain diets. Journal of Animal Science, v. 27 p.1350-1356, 1968.

FRAPE, D. Equine nutrition and feeding. 2.ed. Oxford: Blackwell Science Ltda. 1998. 564p.

GALZERANO, L.; BRETAS, A. A.; MORGADO, E.; ALMEIDA, F.Q.; Balanço hídrico e balanço de nitrogênio em equinos alimentos com diferentes dietas. Revista Electrónica de Veterinária - REDVET. v.7, n.11, 2006. Disponível em: <http://www.veterinaria.org/revistas/red$\mathrm{vet} / \mathrm{n} 101006 . \mathrm{html}>$

GOER, R.J.; MCCUTCHEON, L.J.; ECKER, G.L.; LINDINGER, M.I. heat storage in horses during submaximal exercise before and after humid heat acclimation. Journal of Applied Physiology, v.89, p. 2283 - 2293. 2000.

GROENENDYK, S.; ENGLISH, P.B. External balance of water and electrolytes in the horse. Equine Veterinary Journal, v.20, p.189-193, 1988.

KRISTULA, MA, MCDONNELL, SM. Drinking water temperature affects consumption of water during cold winter weather in ponies. Applied Animal Behavior Scince; v.41 p.155-160, 1994.

LEWIS, L.D. Nutrição Clínica Equina: Alimentação e Cuidados. São Paulo, Ed. Roca, 2000. 710p.

MCDONNELL, S.M.; FREEMAN, D.A.; CYMBALUK, N.F.; SCHOTT, H.C. Behavior of stabled horses provided continuous or intermittent access to water. Animal Journal Veterinary Review, v.60 p.1451- 1456, 1999.

MCDONNELL, S.M.; KRISTULA, M.A. No effect of drinking water temperature (ambient vs. chilled) on consumption of water during hot summer weather in ponies. Applied Animal Behavior Science, v. 41p.155-160, 1996.
NATIONAL RESEARCH COUNCIL - NRC, 1989. Nutrient Requirements of Horses, 5th revised, Washington DC. $1989.112 \mathrm{p}$.

NATIONAL RESEARCH COUNCIL - NRC, 2007. Nutrient Requirements of Horses, 6th revised, Washington DC. 2007. 341p.

NYMAN, S.; DAHLBORN, K. Effect of water supply method and flow rate on drinking behavior and fluid balance in horses. Physiologic Behavior, v.73, p.1-8, 2000.

OLIVEIRA, C.A.A.; ALMEIDA, F. Q.; VIEIRA, A. A.; LANA, A.M.Q.; MACEDO, R.; LOPES, B.A. Cinética de Passagem da Digesta, Balanço Hídrico e de Nitrogênio em Eqüinos Consumindo Dietas com Diferentes Proporções de Volumoso e Concentrado. Revista Brasileira de Zootecnia, v.32, n.1, p.140-149, 2003.

PEARSON, R.A.; MERRIT, J.B. Intake, digestion and gastrointestinal transit in resting donkeys and ponies and exercised donkeys given ad libitum hay and straw diets. Equine Veterinary Journal, v.23, n.5, p.339-343, 1991.

RIBEIRO, L.B.; ARRUDA, A.M.V.; PEREIRA, E.S.; TONELLO, C.L.; BARRETO, J.C. Avaliação do consumo de nutrientes e água por equinos alimentos com dietas contendo diferentes subprodutos agroindustriais. Revista da Faculdade de Zootecnia, Veterinária e Agronomia FZVA, Uruguaiana, v.16, n.1, p. 120-133. 2009.

SILVA, J.D. Análises de Alimentos: Métodos Químicos e Biológicos, Imprensa Universitária, Viçosa, UFV, 2002. 235 p.

UNIVERSIDADE FEDERAL DE VIÇOSA - UFV. Sistema de análises estatísticas e genéticas - SAEG. Versão 9.1. Viçosa, MG, 2008. (CD-ROM).

VAN SOEST, P.J.; ROBERTSON, J.B.; LEWIS, B.A. Methods for dietary fiber, neutral detergent fiber and non starch polysaccharides in relation to animal nutrition. Journal of Dairy Science, v.74, p.3583-3597. 1991. 